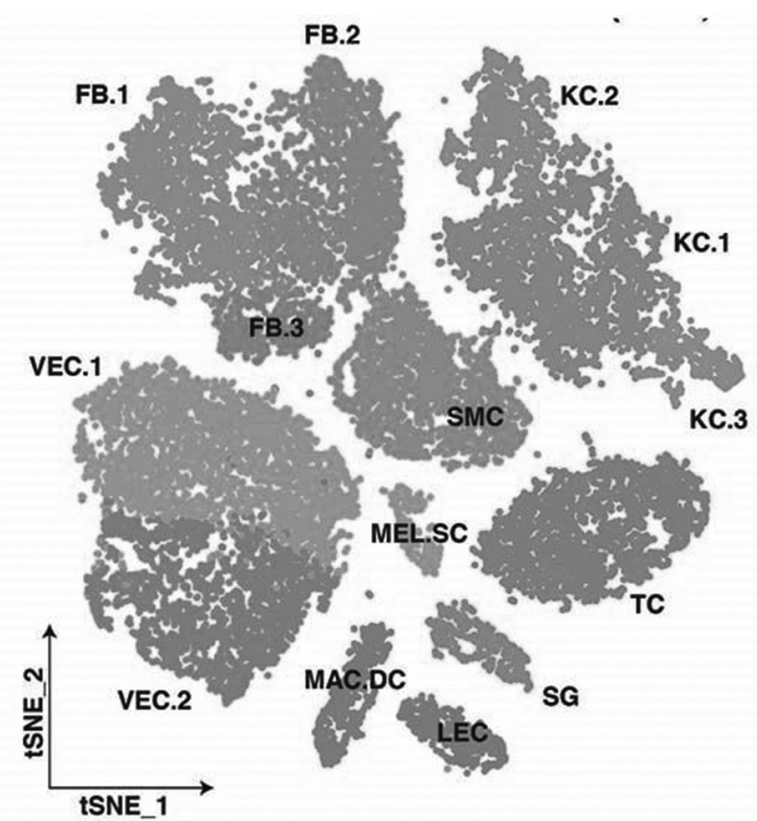

Abstract 194 Figure 1 Major clusters of cells derived from 8 patient skin punch biopsies visualized by tSNE $(n=7280)$. Differential gene expression analysis guided by established lineage markers revealed three keratinocyte clusters (KC1-KC3), two fibroblast clusters (FB1, FB2), smooth muscle cells (SMC), two endothelial cell clusters (VEC, LEC), melanocytes (MEL), sweat gland cells (SG), macrophages/dendritic cells (MAC-DC) and T cells (TC). Ranked by abundance, patient skin exhibited $\mathrm{KC}>\mathrm{FB}>\mathrm{EC}>\mathrm{MAC}-\mathrm{DC}>\mathrm{SMC}>\mathrm{TC}>\mathrm{SG}>\mathrm{MEL}$

Conclusions Single-cell RNAseq is both feasible and informative in cell-specific transcriptome analysis of fresh non-lesional non-sun exposed LN skin biopsies. 10X genomics significantly increases cell numbers and facilitates identification of major cell clusters compared to Fluidigm C1. The expression of fibroblasts and genes reflective of fibrotic and interferonrelated pathways support the application of this approach to study readily accessible tissue for biomarkers related to disease progression.

Funding Source(s): Judith and Stewart Colton Center for Autoimmunity at NYU Langone Health

Differential gene expression analysis guided by established lineage markers revealed three keratinocyte clusters (KC1KC3), two fibroblast clusters (FB1, FB2), smooth muscle cells (SMC), two endothelial cell clusters (VEC, LEC), melanocytes (MEL), sweat gland cells (SG), macrophages/dendritic cells (MAC-DC) and T cells (TC). Ranked by abundance, patient skin exhibited $\mathrm{KC}>\mathrm{FB}>\mathrm{EC}>\mathrm{MAC}-\mathrm{DC}>\mathrm{SMC}>\mathrm{TC}>\mathrm{SG}>\mathrm{MEL}$.

\section{SEQUENTIAL BLOCKADE OF OX40L FOLLOWED BY PD-1 ALTERED THE SUPPRESSIVE FUNCTION AND PROLIFERATION OF CD4+ REGULATORY T CELLS IN LUPUS MICE}

${ }^{1}$ Maida Wong*, ${ }^{2}$ Bevra H Hahn. ' University of California Irvine/VA Healthcare System Long Beach; ${ }^{2}$ University of California, Los Angeles

\subsection{6/lupus-2019-Ism.195}

Background In systemic lupus erythematosus (SLE), dysregulated production of autoantibodies is a consequence of disrupted $\mathrm{T}$ cell homeostasis. We have published that blockade of
Programmed death-1 (PD-1), a negative regulator in $\mathrm{T}$ cells, limits helper $\mathrm{T}$ cell $(\mathrm{Th})$ activation, restores regulatory $\mathrm{T}$ cell (Treg) suppression, and reinstates immune cell function. We have also shown that attenuated PD-1 expression in Treg down-regulates OX40L, which helps restore the suppressive capacity of Treg. However, concurrent administration of different immunotherapies may negate positive outcomes. We hypothesize that the sequence of blocking PD-1 and OX40L influences the induction and sustainability of Treg suppressivity. Methods We treated 8-week-old BWF1 mice with a neutralizing $\mathrm{Ab}$ against $\mathrm{PD}-1$ or $\mathrm{OX} 40 \mathrm{~L}$ intraperitoneally. At age 25 weeks when anti-dsDNA began to rise, mice were treated with either anti-PD1 or anti-OX40L that they had not received at age 8 weeks. OX40L, Foxp3 and PD-1 expression on CD4 +CD25+Treg from spleens, apoptosis of Treg and CD4 +CD25 Th were measured by flow cytometry. Seum production of IFN (Th1), IL4 (Th2), IL17a (Th17) and TGF(Treg), and anti-dsDNA (B cells) were measured by ELISA. The survival of these mice were compared to those treated with anti-PD1 alone at 8 weeks, which we previously demonstrated prolonged survival with delayed onset of proteinuria. Results Anti-OX40L suppressed Th function and proliferation independent of Foxp3 expression in Treg with decreased antidsDNA production. Subsequent blockade of PD-1 in antiOX40L-treated mice generated more PD1loTreg with increased TGF- production; it sustained Treg suppressivity and delayed onset of proteinuria when compared to mice treated with anti-PD1 alone. Conversely, sequential blockade of antiOX40L in anti-PD1-treated mice did not promote Treg survival and their disease inconsistently progressed: these mice had predominantly PD1hi or PD1-Treg, and antagonistic OX40L could not restore their suppressivity.

Conclusions Effective induction of Treg is associated with low expression of PD-1 and OX40L, which permits Treg to survive and perform cell suppressive function. Combination of Abs targeting OX40L and PD-1 can improve Treg function and survival outcomes, but it is determined by the timing and sequence of $\mathrm{Ab}$ administration: blocking OX40L followed by PD-1 has an additive effect which is not observed when the order of Abs given was reversed. OX40L and PD-1 signaling communicate sequentially with Treg to regulate its suppressive capacity and survival to achieve peripheral tolerance in SLE, suggesting that treatment with one immunotherapy could change the biology of T-cell signaling such that another immunotherapy may lose its efficacy or has unexpected negative outcome.

Funding Source(s): None

\section{IMMUNE PATHOLOGY CAUSED BY INTRINSIC LOSS OF CONTROL IN CD4 T CELLS}

Jeroen Roose*. UCSF, Department of Anatomy

10.1136/lupus-2019-Ism.196

Background Naïve T cells constantly see self, need to avoid spontaneous immunity, yet be primed to react to foreign antigens when an infection occurs. Both CD4 +and CD8+priimary $\mathrm{T}$ cells exhibit tonic signaling (Myers et al., 2017b) and continuous interactions of the TCR with self-p/MHC generate tonic signals (Stefanova et al., 2002). T cells lacking the central adapter molecule LAT cause a spontaneous lymphoproliferative $\mathrm{T}$ helper 2 (TH2) cell syndrome in mice. Thus, LAT 


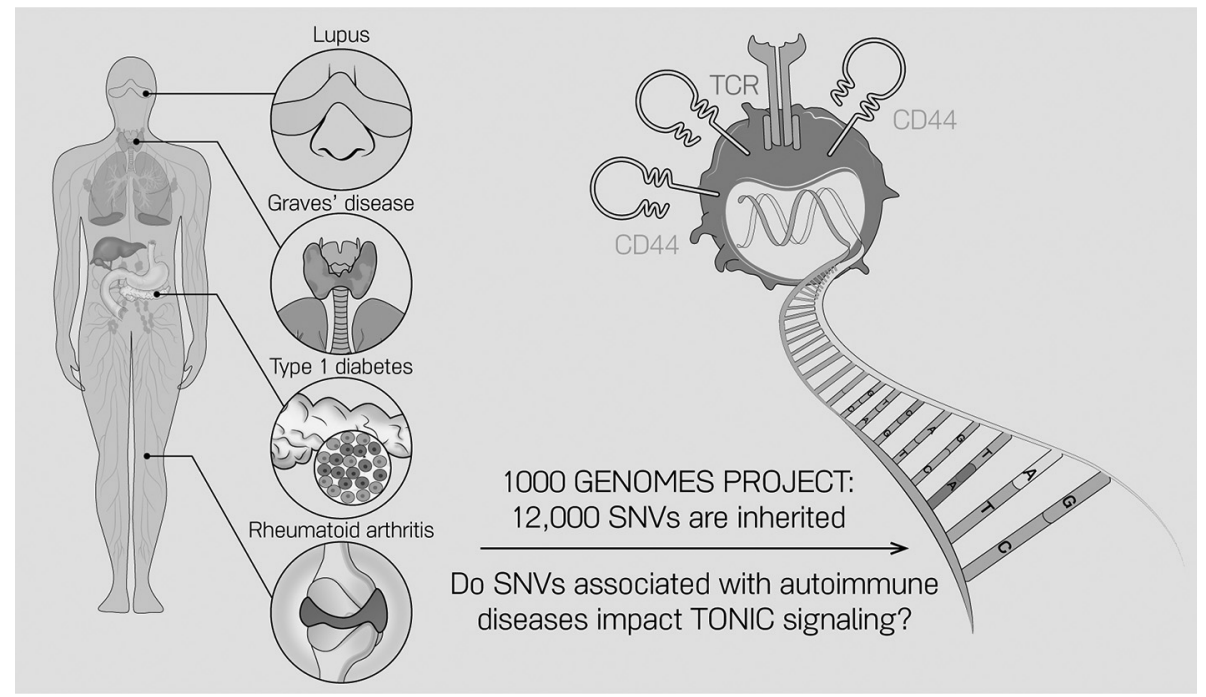

Abstract 196 Figure 1 SNVs and Tonic Signals in T cells

constitutes an unexplained maintenance cue. Our studies of a mouse model with a single nucleotide variant in Rasgrp1, Rasgrp1Anaef, and autoimmune features suggested that basal mTOR signals impact the resting state of $\mathrm{T}$ cells in vivo (Daley et al., 2013). We set out to investigate if tonic signals in primary $\mathrm{T}$ cells establish a primed yet controlled state.

Methods A sophisticated mouse model allowing for inducible deletion of the adapter LAT in T cells was coupled to quantitative biochemical analyses of primary $\mathrm{T}$ cells, gene expression analyses, and functional $\mathrm{T}$ cell assays, Through our Rasgrp1Anaef mouse model, quantitative biochemical analyses of primary $\mathrm{T}$ cells, and genome-wide ribosome profiling, we established that tonic mTORC1 signals shape the baseline translational landscape in resting $\mathrm{T}$ cells in vivo.

Results In 2017, we described how tonic signals prevent aberrant basal activity of naïve T cells; Tonic signals through LAT (Linker for activation of $\mathrm{T}$ cells) and the transcriptional regulator HDAC7 maintain mRNA expression of a cluster of target genes that are negative regulators of spurious $\mathrm{T}$ cell proliferation and Th2 differentiation (Myers et al., 2017a).

We recently discovered robust and selective mTORC1 kinase signals in resting, naive $\mathrm{CD} 4+\mathrm{T}$ cells. We find that RasGRP1 is necessary and sufficient to generate tonic mTORC1 signals. These tonic mTORC1 signals govern a baseline translational program and impact the capacity of CD4 + T cells to take on effector functions (Myers et al., in revision). Aberrantly increased tonic mTORC1 signals in a Rasgrp1Anaef mouse model (Daley et al., 2013) drive immune pathology, alter the ribosome profiles of resting $\mathrm{T}$ cells, and enhance differentiation to the Tph, Tfh, and Th2 fates.

Conclusions Our fundamental studies on tonic signals demonstrate a dynamic balance in naive $\mathrm{T}$ cells with an inhibitory arm regulated through tonic gene expression and a stimulatory arm by shaping a basal translational landscape that includes a mTOR signature. Dysregulation of mTORC1 signaling has been implicated in autoimmune diseases and $\mathrm{T}$ cells from systemic lupus erythematosus (SLE) patients exhibit mTORC1 activation (Perl, 2016). Single nucleotide- and splice- variants of Rasgrp1 have been implicated in SLE (Yasuda et al., 2007), and Type 1 Diabetes and Graves disease (Plagnol et al., 2011; Qu et al., 2009). The mTORC1 inhibitor rapamycin can block $\mathrm{T}$ cell activation in SLE patients and has therapeutic efficacy in SLE (Perl, 2016). We believe that our fundamental studies provide a novel platform for investigating tonic signal activity in $\mathrm{T}$ cells from autoimmune patients and will discuss latest progress.

Funding Source(s): NSF-GRFP (1650113 to DRM) and the NIH-NIAID (R01-AI104789 and P01-AI091580 to JPR).

Are altered tonic signals in $\mathrm{T}$ cells a common consequence of single nucleotide variants (SNVs)?

\section{RACIAL DISPARITIES IN LUPUS MEDICATION ADHERENCE}

Kai Sun*, Amanda M Eudy, Jennifer L Rogers, Lisa G Criscione-Schreiber, Jayanth Doss, Rebecca E Sadun, Megan EB Clowse. Duke University

\subsection{6/lupus-2019-Ism.197}

Background Medication non-adherence is common among systemic lupus erythematosus (SLE) patients and is disproportionately higher among minorities. We aimed to examine the adherence gap between minority and Caucasian SLE patients and explore factors associated with non-adherence that may present avenues for intervention.

Methods Cross-sectional data were obtained via survey and chart review from consecutive SLE patients with 1 scheduled lupus medication. The Medication Adherence Self-Report Inventory (MASRI) was used to estimate adherence in the preceding month from 0\%-100\%. High Self-reported Adherence was defined as MASRI 90\%. Pharmacy refill data in the preceding 3 months were obtained by phone calls. High Refills was defined as a medication possession ratio of $80 \%$ for all prescribed SLE medications. Additional covariates included: positive and negative affect, self-efficacy, patient-reported health status, patient-rated interactions with providers, rheumatic medication regimen complexity, SLE Disease Activity Index (SLEDAI), and physician global assessment for inflammatory (PGA1) and noninflammatory (PGA2) symptoms. Adherence groups were compared.

Results 84 enrolled (37\% Caucasians, 59\% African American, $1 \%$ Native American, and 4\% Hispanic). Median age was 42 (range 22-68), 95\% were female, 49\% had college education, and $49 \%$ had private insurance. 54 (64\%) had High Self- 